\section{RNA interference meets brain disease}

Mouse models are essential tools in the quest to understand human diseases, but fine spatial and temporal control of gene expression are difficult to achieve with traditional transgenic and knockout techniques. The potential of post-transcriptional gene silencing, or RNA interference (RNAi), to overcome these problems in the mammalian brain has been explored by Ralph DiLeone and his team from The University of Texas Southwestern Medical Center at Dallas. Their data, published in the December issue of Nature Medicine, show that RNAi-mediated knockdown of the dopaminesynthesis enzyme tyrosine hydroxylase in the midbrain generates mice with a Parkinson's disease-like phenotype.
RNAi is based on a highly conserved defense mechanism in which short, double-stranded RNA fragments are enzymatically snipped from foreign genetic material and subsequently disrupt the translation of their messenger RNA of origin. DiLeone et al. designed hairpinshaped RNA fragments to specifically target two unique 24-nucleotide stretches of the tyrosine hydroxylase mRNA. These fragments were co-expressed with enhanced green fluorescent protein (EGFP) in an adeno-associated viral vector, which was then injected into the midbrain of adult mice.

Twelve days after injections to the substantia nigra pars compacta, immunostaining and quantitative real-time reverse transcription-

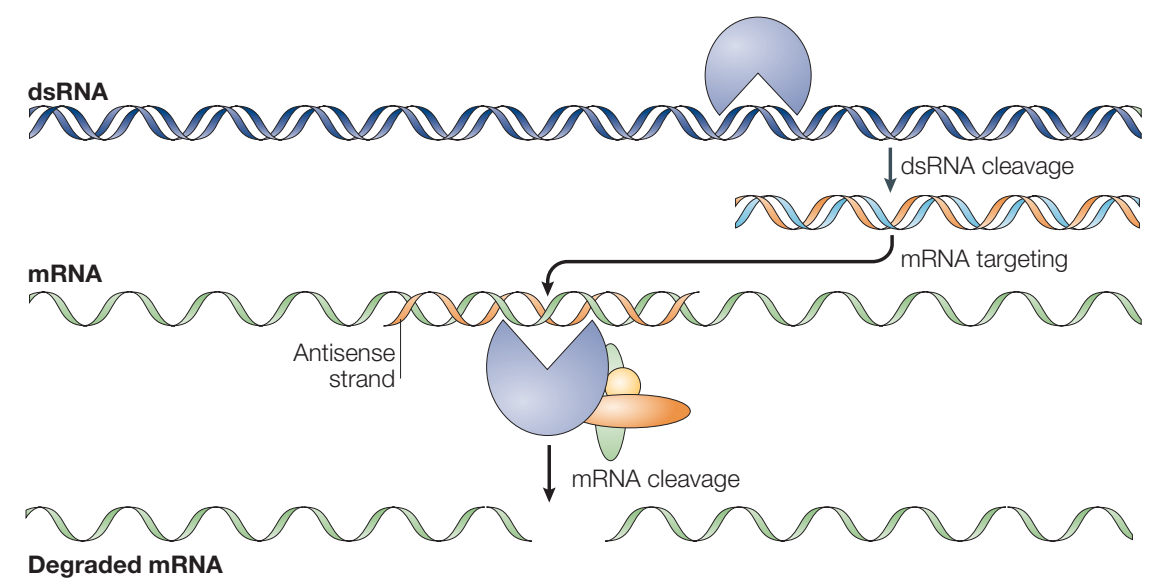

polymerase chain reaction showed that expression of tyrosine hydroxylase was markedly reduced in infected dopamine neurons (that is, those neurons positive for both EGFP and dopa-decarboxylase, a marker of dopamine neurons). This attenuation of expression persisted for at least 50 days. Levels of tyrosine hydroxylase in regions of the nucleus accumbens innervated by infected midbrain neurons were also reduced. By contrast, high levels of tyrosine hydroxylase expression were maintained in control neurons infected with a vector containing 'scrambled', non-tyrosine hydroxylase-specific short RNA fragments.

Importantly, interfering with tyrosine hydroxylase expression correlated with impaired motor function, as assessed by the rotarod test and a reduced response to amphetamine. This phenotype mimics that of neurotoxin-induced models of Parkinson's disease that show neurodegeneration of the substantia nigra, indicating that loss of dopamine in this brain region is sufficient to induce aberrant motor behaviour. So, DiLeone and colleagues succeeded in developing a potentially useful model for studying Parkinson's disease, while demonstrating the general utility of RNAi as a fast and effective means of probing the molecular mechanisms of disease.

Suzanne Farley

\section{(D) References and links \\ ORIGINAL RESEARCH PAPER Hommel, J. D. et al. Local gene knockdown in the brain using viral-mediated RNA interference. Nature Med. 9, 1539-1544 (2003) FURTHER READING Opalinska, J. B. \& Gewirtz, A. M. Nucleic- acid therapeutics: basic principles and recent applications. Nature Rev. Drug Discov. 1, 503-514 (2002) WEB SITE \\ Encyclopedia of Life Sciences: http://www.els.net/} Parkinson disease

\title{
Protecting against hypoglycaemia
}

Hypoglycaemia is a common complication in insulin-dependent diabetes, and it can lead to brain damage and long-term cognitive impairment. A study published in the Journal of Neuroscience points towards a potential therapeutic target for treating patients with severe hypoglycaemia.

Hypoglycaemia seems to cause neuronal damage by initiating a cascade of events that includes a large increase in extracellular glutamate in the brain. Sustained activation of glutamate receptors leads to excitotoxicity, DNA damage and cell death, and also causes activation of poly(ADPribose) polymerase 1 (PARP1), which is involved in DNA repair. Extensive activation of PARP1 can lead to cell death, and this is thought to be an important mediator of glutamate toxicity.

Suh et al. found that cortical cultures derived from PARP1-knockout mice, or cultures treated with a PARP1 inhibitor, were largely resistant to hypoglycaemic neuronal death. And in a rat model of hypoglycaemia produced by injection of insulin, a PARP1 inhibitor reduced neuronal damage in the cortex and hippocampus by up to $80 \%$ - even if given as late as two hours after the hypoglycaemic episode.
As well as protecting the vulnerable neurons against hypoglycaemic damage, the PARP1 inhibitor also prevented the cognitive dysfunction (in particular, deficits in spatial learning and memory) that was seen in untreated animals after hypoglycaemia. At present, the only treatment for hypoglycaemia in humans is to raise blood glucose by giving glucose or the hormone glucagon, but this does not prevent ongoing brain damage. If PARP1 inhibitors could be shown to work in humans, even when given after normalization of blood glucose levels, they would be a valuable addition to the medical toolbox.

Rachel Jones

(2) References and links ORIGINAL RESEARCH PAPER Suh, S. W. et $a$. Hypoglycemic neuronal death and cognitive impairment are prevented by poly(ADP-ribose) polymerase inhibitors administered after hypoglycemia. J. Neurosci. $\mathbf{2 3}$, 10681-10690 (2003) 